\title{
Interview with Edward Witten
}

Hirosi Ooguri

\begin{abstract}
This is a slightly edited version of an interview with Edward Witten that appeared in the December 2014 issue of Kavli IPMU News, the news publication of the Kavli Institute for the Physics and Mathematics of the Universe (IPMU). An abridged Japanese translation has also appeared in the April and May 2015 issues of Sugaku Seminar, the popular mathematics magazine. The interview is published here with permission of the Kavli IPMU.

The interview took place in November 2014 on the occasion of Witten's receipt of the 2014 Kyoto Prize in Basic Sciences. Witten is Charles Simonyi Professor in the School of Natural Sciences at the Institute for Advanced Study in Princeton. Yukinobu Toda and Masahito Yamazaki, two junior faculty members of the Kavli IPMU, also participated in the interview.
\end{abstract}

Ooguri: First I would like to congratulate Edward on his Kyoto Prize. Every four years, the Kyoto Prize in Basic Sciences is given in the field of mathematical sciences, and this is the first prize in this category awarded to a physicist.

Witten: Well, I can tell you I'm deeply honored to have this prize.

Ooguri: It is wonderful that your work in the area at the interface of mathematics and physics has been recognized as some of the most important progress in mathematics as well as in physics. For those of us working in this area, this is also very gratifying.

Witten: Actually, in my acceptance speech a couple of days ago, I remarked that I regard it also as a recognition of the field, not just of me.

\section{Generalizing Chern-Simons}

Ooguri: This conversation will appear as an article in the Japanese magazine, Sugaku Seminar, as

Hirosi Ooguri is Fred Kavli Professor of Theoretical Physics and Mathematics, the director of the Walter Burke Institute for Theoretical Physics, and the deputy chair of the Division of Physics, Mathematics and Astronomy at the California Institute of Technology. He is also a principal investigator of the Kavli Institute for the Physics and Mathematics of the Universe at the University of Tokyo. His email address is ooguri@theory.caltech.edu.

All photos provided by and used with permission of Kavli IPMU.

DOI: http://dx.doi.org/10.1090/noti1248 well as in the Kavli IPMU News. You have already given two interviews for Sugaku Seminar. In 1990, at the International Congress of Mathematicians in Kyoto, you received the Fields Medal. On that occasion, Tohru Eguchi had an interview with you. You also had a discussion with Vaughan Jones, another Fields Medalist at the congress, and I remember you expressed interest in generalizing your work in the Chern-Simons theory with a spectral parameter, which is very natural from Edward Witten. the point of view of integrable models.

Witten: Yes, I very much wanted to find an explanation along these lines of the "integrability" that makes it possible to get exact solutions of two-dimensional lattice models such as the Ising model. I was completely unsuccessful, but just in the last couple of years, something in the spirit of what I wanted to do was done by Kevin Costello.

Ooguri: We were just talking about Costello's work before you arrived here. Do you think that it achieved what you wanted to do at that time?

Witten: Yes. Integrable models have many facets, and there is no one way to understand everything. 
But I would say that specifically the kind of explanation I was looking for is what Costello found. What Costello did involves a very simple but beautiful twist on the three-dimensional ChernSimons theory, in which he simply replaced one of the three real dimensions of space by a complex variable $z$.

Ooguri: It means going to four dimensions.

Witten: This is a four-dimensional world with two real coordinates and a complex coordinate $z$. Costello defined a 4-form, which was the wedge product of the Chern-Simons 3 -form with the 1 -form $d z$. He studied this as the action of a fourdimensional theory. There is a crucial technical detail: for this theory to make any sense, the differential operator that is obtained by linearizing the equations of motion must be elliptic modulo the gauge group. I think that this is a little surprising, but it is true. And given that, he then has a generalization of the Chern-Simons theory that does not have the full three-dimensional symmetry, but it does have a complex variable, namely $z$.

If you think carefully, you will see that integrability, the Yang-Baxter equation, involves two-dimensional symmetry but not really threedimensional symmetry. The reason I was unable to incorporate the spectral parameter was that I was working in the context of three-dimensional topological field theory. In three-dimensional topological field theory, in addition to the moves where knots cross - that is, in addition to the Yang-Baxter relation-you have further relations involving creation and annihilation. There are Reidemeister moves that are valid in topological field theory but are not relevant for integrable systems. I couldn't find the spectral parameter because I was trying to use topological field theory. Costello made a very simple twist, replacing a real variable by a complex variable, and then everything worked beautifully. I definitely regard that as the explanation I was trying to find, unsuccessfully, around 1990.

Ooguri: I see. So, after twenty-three years, finally your question was answered.

At the time of your second visit to Kyoto in 1994, you were just finishing your work on the SeibergWitten theory and also the Vafa-Witten theory. I remember a discussion session we had at the Research Institute of Mathematical Sciences, Kyoto University, with you and Hiraku Nakajima, where Nakajima explained his work on the action of an affine Lie algebra on the cohomology of the moduli space of instantons. In the second interview you had with Tohru Eguchi for Sugaku Seminar, you mentioned progress in mirror symmetry and S-duality at the time and expressed a hope of a more unified view on duality encompassing gauge theory and string theory. Some of this hope has been achieved in the last twenty years, I think.
Witten: Definitely some of it has been achieved. One thing that was achieved in the couple of years after that second interview was simply that there emerged a picture of nonperturbative dualities in string theory, generalizing what happens in field theory. However, there are other aspects that are still mysterious and not clearly understood.

On the bright side, the fact that four-dimensional gauge dualities and a lot of dualities in lower dimensions come from the existence of a sixdimensional conformal field theory is a major insight in understanding dualities better. We haven't gotten to the bottom of things because we don't really understand the six-dimensional theory, but just knowing that the matter should be understood in terms of the properties of the sixdimensional theory is an advance in understanding duality that certainly wasn't there at the time of this last interview.

\section{Was a Skeptic about Duality}

Ooguri: I should introduce our discussants today. Yukinobu Toda is a mathematician and an associate professor at the Kavli IPMU, and he received his $\mathrm{PhD}$ in 2006. Masahito Yamazaki is a physicist and a new assistant professor at the Kavli IPMU, and he received his $\mathrm{PhD}$ in 2010. They represent the young generation of mathematicians and physicists working at the interface of string theory and gauge theory.

At your Kyoto Prize Commemorative Lecture, you reviewed your career in theoretical physics. You entered graduate school in 1973, when the asymptotic freedom was just theoretically being discovered. You came to Japan the second time in 1994 and gave the interview we were just talking about, and it was roughly twenty years after you started in graduate school. Another twenty years have passed since that time, so I thought we should start by trying to catch up with the second twenty years of your career and see what your thoughts have been on some of the most important progress.

We have already started to talk about some of the developments since the interview in 1994, but maybe you can expand on that and tell us what you think have been the highlights in this area in the last twenty years.

Witten: Certainly one of the highlights has been the understanding of nonperturbative dualities in string theory, as a result of which we have a much wider picture of what string theory is. In 1994 we knew about mirror symmetry and other two-dimensional dualities that arise in string perturbation theory, and we were really just beginning to think that there are similar dualities in space-time: four-dimensional gauge theory dualities that are analogous to the twodimensional dualities. But in 1994 it was really just 
a guess that something analogous might happen in string theory.

By this time there were clues in the literature, and a number of new ones had been discovered in the early 1990s. The clue that influenced me the most was the work of John Schwarz and Ashoke Sen, who showed that the low-energy effective action of the heterotic string on a six-torus had properties consistent with the existence of a nonperturbative $S L(2, Z)$ duality. They didn't have what I regarded as really decisive evidence for that conjecture, but their ideas were very suggestive.

It still was not clear to me how one could find decisive evidence for nonperturbative dualities in space-time. At least to me, the first such evidence appeared in a short but brilliant paper by Ashoke Sen on a two monopole bound state in $N=4$ super Yang-Mills theory. To me, that was fundamentally new evidence for the Montonen-Olive duality conjecture. It convinced me that the duality had to be right and, equally important, it convinced me that it was possible to understand it better.

Ooguri: I thought that Sen's paper gave strong evidence for the S-duality, but it was your paper with Vafa that convinced us.

Witten: Thank you. Sen's paper showed that you could actually go well beyond the suggestive but somewhat limited arguments about electricmagnetic duality that had been known and learn something fundamentally new. Until Sen's paper, I had felt that what we understood about electricmagnetic duality, even the work of Sen and Schwarz, which had definitely influenced me, was in the framework of what Montonen and Olive had understood twenty years before. But Sen did a simple and elegant calculation, finding a bound state of two monopoles whose existence was predicted by the duality. That inspired me to believe that one could do more.

With this inspiration, and trying to find more evidence for the duality conjectures, Cumrun Vafa and I started to study the Euler characteristics of instanton moduli spaces. It was not too hard to see that electric-magnetic duality of supersymmetric Yang-Mills theory implied that the generating function of those Euler characteristics should be a modular function. Luckily for us, mathematicians in a number of cases-and this includes the work of Nakajima that you mentioned beforehad computed these Euler characteristics or had obtained closely related results from which the Euler characteristics could be understood. We found that the expected modularity held in all cases. (In one case-the four-manifold $\mathbb{C P}^{2}-$ we ran into a "mock modular form," a concept that was new to us at the time but has made many subsequent appearances in gauge theory and string theory.)
Also during this period, Nathan Seiberg had been using holomorphy as a tool to analyze the dynamics of supersymmetric gauge theories. He wanted to understand what happened in $N=2$ theories. We started talking about it, and the Sen paper inspired us to think that duality would play a role. That was one of the clues that actually led to our work on what became Seiberg-Witten theory.

Ooguri: It may be hard to believe for young people like Masahito Yamazaki or Yukinobu Toda, but before 1994 S-duality, at least for me, was something very hard to believe. It was like a beautiful dream. It would be nice to have, but you cannot realistically hope that something like that could possibly happen. As I said, the first evidence was Sen's paper, and in some sense Edward's work with Vafa nailed it. After that, everybody believed it.

Masahito Yamazaki: That's surprising, because I thought that the paper of Claus Montonen and David Olive is quite old. Were people skeptical about the idea?

Witten: This might make you laugh, but I'll tell you my early history with the Montonen and Olive paper. First of all, I hadn't heard of it until I was visiting Oxford at the end of 1977. Michael Atiyah showed this paper to me and said I should go to London to discuss it with Olive. So, I looked at the paper and got in touch with David Olive and arranged to visit him. But by the time I got to London, I was pretty skeptical. Have you looked at their original paper?

Masahito Yamazaki: Yes, I have.

Witten: In their original paper, they considered a bosonic theory of a gauge field and a real scalar (valued in the adjoint representation). They assumed that the potential energy for the scalar field is identically zero, and they found remarkable formulas for particle masses that are valid precisely in this case. Their proposal of electric-magnetic duality was based on the fact that their mass formula was symmetrical between electric and magnetic charge.

However, I knew quantum field theory well enough to know that saying that the potential energy for the scalar field is zero is not a meaningful statement quantum mechanically. If it were, we would not have a gauge hierarchy problem in particle physics. So, by the time I got to London to see Olive, I definitely was a skeptic. But since I was there to see him, I didn't want to just say that his idea was nonsense. We tried to make sense out it. So we discussed it in the context of supersymmetry, simply because with supersymmetry the mass renormalization (and even the full effective potential) of a scalar can be zero. This was the only context in which it seemed to me that the brilliant idea of Montonen and Olive could make sense. By the end of the 


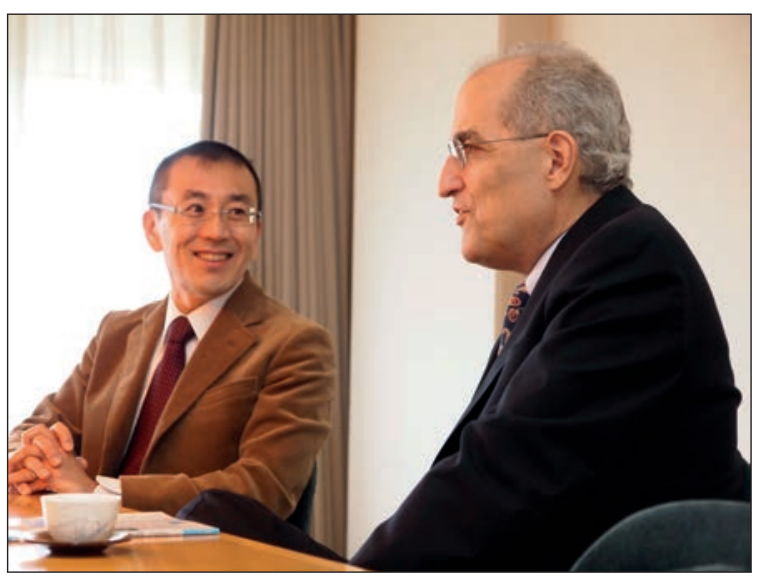

Ooguri and Witten.

day, we found that their formulas are valid in the context of $N=2$ supersymmetry. So we wrote a paper on that, and it was quite a satisfying paper to write, but I drew the wrong conclusion from that paper. The conclusion I drew was that we had explained their formulas without needing to assume nonperturbative duality.

Ooguri: Right. That was the message I got by reading your paper with Olive, too. The seemingly miracle phenomenon was explained simply by supersymmetry.

Witten: So at the time, and for many years after, I felt that there was not really a lot of evidence for nonperturbative duality in four dimensions.

Thus, to return to Masahito's question, I was a skeptic about electric-magnetic duality during these years, but actually I was a skeptic on two levels. One, I was skeptical that it was true, and two, I was skeptical that you could really say anything about it even if it were true.

To give a more complete picture, in the early 1990s there were various novel clues, some from the work of people like Mike Duff, and also Curt Callan, Jeff Harvey, and Andy Strominger, studying solitons in string theory, and then also there was the work of Sen and Schwarz that I already mentioned. I remember that at the Strings 1993 meeting in Berkeley, John Schwarz was more excited than I had ever seen him since January 1984. In January 1984, telling me about his latest work with Michael Green, he said, "We are getting close," but I didn't understand what he thought he was getting close to. It turned out, however, that that was a few months before they canceled the anomalies. When John was so excited at the Strings meeting at Berkeley, I decided that I had better take him seriously.

If you had looked at it with the same skepticism I had had since the Montonen and Olive paper, you would have said that Sen and Schwarz were just discussing low energy physics and did not have solid evidence about strong coupling behavior. But John's enthusiasm put enough of a dent in my skepticism that I started looking more closely at the papers of Duff and other authors on solitons in string theory. At some point, I think in the fall of 1993, Duff sent me an assortment of his papers, and I took them to heart. I don't remember right now all of the papers on solitons in string theory that I looked at during this period, but certainly one important one was by Callan, Harvey, and Strominger.

There is another part of the background to this period that I should explain. Mike Duff, Paul Townsend, and other physicists working on supermembranes had spent a couple of years in the mid-1980s saying that there should be a theory of fundamental membranes analogous to the theory of fundamental strings. That wasn't convincing for a large number of reasons. For one thing, a three-manifold doesn't have an Euler characteristic, so there isn't a topological expansion as there is in string theory. Moreover, in three dimensions there is no conformal invariance to help us make sense of membrane theory; membrane theory is nonrenormalizable just like general relativity.

There are all kinds of technical objections, but at some point around 1990 or 1991, instead of trying to think of membranes as fundamental objects, people working in this area started thinking of membranes and other $p$-branes as nonperturbative objects that might exist in string theory. In general terms, this idea did make sense. In more detail, the situation was more complicated. If you actually looked at the papers, some of them made a lot of sense because they had a classical soliton solution with good properties. (Even then, the solutions usually had unusual properties that in some cases were clues to later discoveries.) Other papers made a little bit less sense, because the classical solution involved a singularity that appeared in a region in which the classical approximation wasn't good. But the idea of membranes as nonperturbative soliton-like objects in string theory made a lot of sense even if the details in some papers were dubious. I was still a bit of a skeptic about what one can do with this idea, but for reasons I've been explaining, I was paying a lot more attention. And that is actually why, when the Sen paper on the two-monopole bound state came out, I was ready to completely change my outlook.

Sen's paper showed that one can do something new about strong coupling, and it was clear that if one had been inspired the way Sen was, one could have done what he did ten or fifteen years before. So, it showed that we had been missing opportunities. That definitely changed the direction in my work. It led to the paper with Vafa that you've been kindly 
mentioning, and it helped put Seiberg and me on the right track for doing what we did in 1994 and then...

Ooguri: This is a great story that shows that chance favors the prepared mind, as Pasteur said. After that, you even went to string dualities.

\section{String Duality Revolution}

Witten: By the end of 1994, we had the experience of nonperturbative dualities in field theory both in two dimensions and in four dimensions. In the two-dimensional case, for example, if one studies a sigma-model with Calabi-Yau target space (such as is important in studying the compactification of string theory), one finds that the quantum theory can be extended far beyond the classical geometry of the Calabi-Yau manifold. One finds a web of phase transitions between different geometrical and nongeometrical descriptions of the sigmamodel, which represent different semi-classical limits of the theory. The Montonen-Olive duality conjecture, as refined by later authors, said that something similar happens in $N=4$ super YangMills theory in four dimensions, and Seiberg and I in 1994 had found something somewhat similar for $N=2$.

Certainly there was a dream that something similar might happen in string theory. Not only was there a dream, but there were a lot of papers in which people had pointed out pieces of such a story. I have already mentioned some of these papers. Another important paper was written by Chris Hull and Paul Townsend in the spring of 1995. They wanted to say that Type IIA superstring theory is the same as M-theory on a circle. The only thing they really didn't do was to try to make it more quantitative. There's a potential contradiction, which is that in Type IIA superstring theory you don't see eleven dimensions. But it turns out, as I realized a little later, that this question has a very simple answer. The eleven-dimensional limit is a region of strong coupling from the point of view of Type IIA superstring theory, and the eleventh dimension isn't visible for weak coupling.

It soon became clear that the same thing was true in other cases. For example, one might hope that Type I superstring theory and the SO(32) heterotic string will be the same. There is an obvious immediate contradiction: the theories have the same massless spectrum and low energy interactions, but beyond the low energy limit they look completely different. The answer is simply that if you match up the low energy field theories, you will find that weak coupling in one is strong coupling in the other.

Once one starts thinking along those lines, it turns out that everything works. What were the implications? This way of thinking certainly led to a more unified picture of what string theory is. But very soon, there were further developments showing that the traditional ways of asking questions were probably inadequate. In the 1980s I was really convinced that in some sense string theory should be based on a Lagrangian that would generalize the Einstein-Hilbert Lagrangian for gravity; it would have a symmetry group which would generalize the diffeomorphism group. So there would be a new classical theory of geometrywith nonperturbative two-dimensional dualities built in as classical symmetries. One would then generate string theory by quantizing this classical theory.

But by the early 1990s, there was a troublesome detail that I personally did not pay much attention to. In the moduli space of Calabi-Yau manifolds, there is a variety of singularities. Some questions involving such singularities had been important in my own work.

Ooguri: You are referring to the work involving linear sigma-models.

Witten: That is correct, and also my work (with Harvey, Vafa, and Lance Dixon) on orbifolds. I had been interested in cases in which the classical geometry has a singularity but the quantum sigmamodel does not; these cases illustrate the difference between ordinary geometry and its generalization in the classical limit of string theory. What I had not taken seriously is that in general, as one deforms the moduli of a Calabi-Yau manifold, one can find singularities of the classical geometry that do also lead to singularities of the corresponding sigma-model.

Such a singularity appears in string theory even in the classical limit, so if you try to interpret string theory as a classical theory that then gets quantized, it looks like the classical theory has a singularity, which is strange. I personally didn't focus on that question, but Strominger explained that such a singularity actually reflects a nonperturbative quantum effect. The singularity arises when a charged black hole becomes massless, and it shows that quantizing a classical theory can't do justice to string theory: there are nonperturbative quantum effects even in what one might have wanted to call the classical limit.

Ooguri: You say there's no analogous result in field theory, this is a genuinely string-theoretic phenomenon.

Witten: I think so.

Ooguri: So, did you think this was evidence that there is no Lagrangian description in string theory?

Witten: It is evidence that you can't fully do justice to string theory in terms of quantizing a classical theory. I don't want to say that there isn't a classical theory, because I believe that from some point of view there is. 
Ooguri: Yes, as an approximate description, but you're saying that you cannot start from classical theory and apply a quantization procedure...

Witten: We can't fully understand string theory by quantizing an underlying classical theory. In some sense it is an intrinsically quantum mechanical theory.

I don't want to say you can't derive string theory by quantizing a classical theory, but you can't fully do justice to it that way, I think.

But let us remember that even in field theory, Montonen-Olive duality means that the same theory has different classical limits, showing that no one classical limit is really distinguished.

Ooguri: But in that case, you have a Lagrangian description.

Witten: Yes, in the Montonen-Olive case, one has a classical Lagrangian, in fact many of them. String theory is a little bit worse because even in what you want to call the classical limit, there are phenomena that you really can't make much sense of from the classical point of view.

Ultimately, Strominger's work illuminated something I'd missed. In the talk I gave at the Strings Conference in 1995 about nonperturbative dualities in string theory, and also in the corresponding paper ["String theory dynamics in various dimensions", arXiv:hep-th/9503124, Nuclear Physics B, volume 443 , issue $1-2$, June 5,1995 , pages $85-126]$, there was one detail that didn't completely make sense. Type IIA superstring theory on a $K 3$ manifold was supposed to be dual to the heterotic string on a four-torus, and in that context I could see that enhanced gauge symmetry resulted from the $K 3$ surface developing an ADE singularity. But an ADE singularity in classical geometry is just an orbifold singularity, and perturbation theory remains valid in string theory at an orbifold. The orbifold does not generate a nonperturbative gauge symmetry. For a few months I was puzzled. Actually, I was making a simple mistake which was corrected by Paul Aspinwall in a paper that he wrote in the summer of 1995. Aspinwall explained the following: In M-theory at an ADE singularity, you have only the hyper-Kähler moduli, but in string theory at an ADE singularity, there also are $B$-field moduli. The conformal field theory becomes singular when the $B$-field moduli are zero; the orbifold describes a nonsingular situation in which the $B$-field moduli are not zero.

When the $B$-field moduli vanish, there is a breakdown of the classical description that's just analogous to what Strominger had shown in his paper on the Calabi-Yau singularity. It leads to enhanced gauge symmetry that, from the standpoint of Type IIA superstring theory, has a nonperturbative origin.
Strominger had considered a charged black hole that arises from a wrapped three-brane, while here the relevant particle is a wrapped two-brane. But the idea is similar.

Ooguri: So this was the beginning of the interaction between the gauge-theoretic idea and the string-theoretic idea where non-Abelian, nonperturbative dynamics of gauge theory can emerge from limits of string theory.

Witten: Right. Another important but extremely simple paper that helped show the implications of string theory for nonperturbative duality in gauge theory was written by Michael Green in 1996. By this time, Joe Polchinski and his collaborators had basically shown that in modern language a system of $n$ parallel branes has $U(n)$ gauge symmetry. I had written a paper at the end of 1995 showing why that was useful, but Green wrote a very simple paper with the following observation. Type IIB superstring theory has a nonperturbative duality symmetry-a fact which we were convinced of by this time-and on the other hand $N=4$ super Yang-Mills theory in four dimensions with gauge group $U(n)$ can arise from a system of $n$ parallel D3branes in Type IIB superstring theory. Combining these two facts and taking the low energy limit, Green was able to deduce the Montonen-Olive duality of $N=4$ super Yang-Mills theory with gauge group $U(n)$. It is simply inherited from the Type IIB superstring theory specialized to the D3-branes. That was an important early example of deducing a gauge theory duality from a string theory duality.

Even before all of this had happened, Mike Duff and Ramzi Khuri in 1993 had written a paper on what they called string/string duality. They had said there should be a self-dual string theory in six dimensions that, looked at in two different ways, would give electric-magnetic duality of gauge theory in four dimensions. It was actually a brilliant idea. The only trouble was they didn't have an example in which it worked.

I realized in mid-1995 that if one took the heterotic/Type II duality on $K 3$ and $T^{4}$ and compactified on another two-torus, one would get an example rather similar to what Duff and Khuri had suggested. They had had in mind self-duality of a string theory, but the example that I considered was a duality between two different string theories. Still, the idea was similar. By the end of 1995, Duff and I and some other authors had an example that followed even more precisely what had been proposed two years before. That involved the $E_{8} \times E_{8}$ heterotic string on a $K 3$ surface with equal instanton numbers in the two $E_{8}$ 's. In all of these cases, one could deduce Montonen-Olive duality from a string theory duality. 
As we're talking, I remember more and more papers from the years 1995-96 that were very dramatic at that time but that were also, honestly, pretty easy to do in most cases. I was reminded of this yesterday by the lecture by Hiraku Nakajima at the Kyoto Prize Workshop. Hiraku started by kindly remembering three lectures I gave at the Newton Institute in 1996. The lectures concerned three papers that I had written (respectively with coauthors Lev Rozansky, Ami Hanany, and Nathan Seiberg). The papers fit together nicely. They were fun to write, and the lectures were also fun to give. But what stands out in my memory is that at that time insights like that were more or less out on the surface. It was quite a fun time to be working in this field. I am hoping that, during my active career, there will be another period like that.

\section{Difference between Knowing What Is True and Why It Is True}

Yukinobu Toda: I am an algebraic geometer. I was originally working on some classical aspects of algebraic geometry, but I became interested in some relationships between algebraic geometry and string theory inspired by your work. You have been discussing S-duality and modular forms. This was surprising from a mathematical point of view-I cannot see why modularity appears. Do you have an insight about that from a mathematical point of view?

Witten: Vafa and I, of course, had a reason, which was the Montonen-Olive duality conjecture. What we did was to show that modularity of a certain generating function of Euler characteristics is a kind of corollary of Montonen-Olive duality. This is somewhat analogous to saying that some statement in number theory follows from the Riemann hypothesis. If somebody shows that something follows from the Riemann hypothesis, one may or may not view this as an explanation of the statement in question, but at least it puts the statement in a bigger framework. Montonen-Olive duality provided an analogous bigger framework in my work with Vafa, and soon afterwards there was a still bigger framework, which was that the Montonen-Olive duality follows from the existence of a certain six-dimensional theory. It also follows in various other ways from string theory dualities, and I have mentioned a few of these constructions. But most physicists would probably say the most complete framework that we have for MontonenOlive duality is its relation to the six-dimension theory.

Ooguri: Yukinobu was asking for some mathematical explanation. At that time, some hint of the mathematical explanation was Nakajima's work about the symmetry of the moduli spaces of instantons. From the mathematical point of view, what the

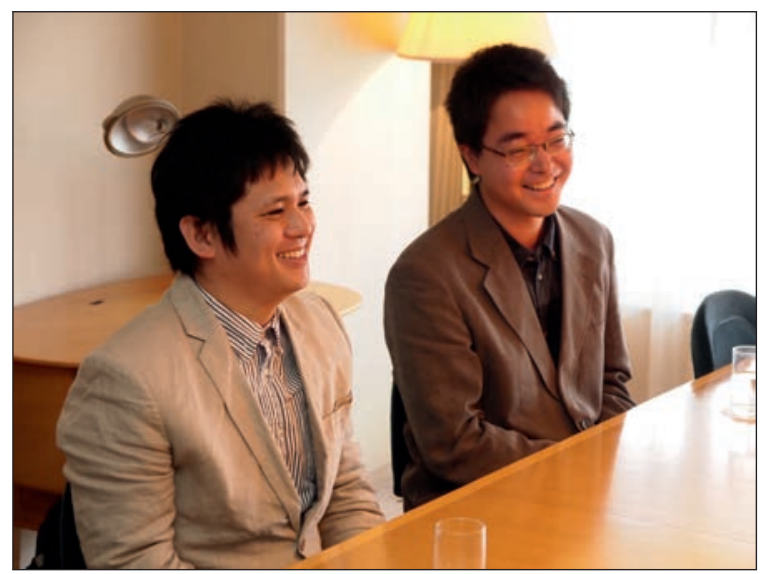

YukinobuToda and Masahito Yamazaki.

Vafa-Witten theory was computing were generating functions of the Euler characteristics of instanton moduli spaces.

Witten: Nakajima's discovery of the affine Lie algebras was a kind of proof and actually a miraculous discovery. But it still leaves one wondering where the affine Lie algebra symmetry came from.

Yukinobu Toda: Right. After computations of the Euler characteristics, we know that it's a modular form, but we don't know why it is modular, even for the simplest example.

Witten: I completely agree. What you're saying is actually something I tried to say in my Commemorative Lecture for the Kyoto Prize. There is a difference between knowing what is true and knowing why it is true. In this case, you have a mathematical proof, but you're still asking why, and physicists ultimately don't know. All that we can do is to offer bigger conjectures, of which this is a manifestation. But we don't really understand the bigger conjectures.

Ooguri: In physicists' perspective, this duality has been geometrized as symmetry in six dimensions.

Witten: But the six-dimensional theory is pretty mysterious.

Yukinobu Toda: Is it not difficult to understand the relationship between $S$-duality and six-dimensional theory?

Ooguri: The relation is very clear, but then you have to make sense of the six-dimensional theory itself.

Witten: We actually know quite a lot about the behavior of the six-dimensional theory, though we do not understand much about how it should be constructed or understood microscopically.

One of the deepest discoveries about the behavior of the six-dimensional theory was made by Juan Maldacena in 1997. He showed that it could be solved for large $N$ in terms of supergravity 
( $N$ refers to the rank of an $\mathrm{SU}(N)$ gauge group). Unfortunately, the regime in which the theory is solved by supergravity isn't the same regime in which we usually have to study it to understand the questions you're asking.

Ooguri: I understand that the large $N$ limit is not S-duality invariant.

Witten: Yes, that is correct. Maldacena's solution of the theory for large $N$ works and makes complete sense. It does not directly help us in understanding Montonen-Olive duality, because it involves studying the theory in a different region of parameters that is not invariant under duality. Or, to put it differently, if one tries to apply Maldacena's solution to understand Montonen-Olive duality, one has to work in a region of parameters in which the description that Maldacena gave is not useful.

But the existence and success of Maldacena's solution definitely increases the confidence of physicists that the six-dimensional theory exists and that all the canonical statements about it are true, even though we don't understand everything. It's a little bit like mathematicians discovering that some new consequences of the Riemann hypothesis are true. This gives one more confidence in the Riemann hypothesis, but it doesn't mean one understands the Riemann hypothesis.

Ooguri: Yukinobu, what is your view on current activities in physics? For example, yesterday Nakajima was saying that it took him eighteen years to understand what Edward was doing in his lectures in Cambridge, and Kenji Fukaya was saying that sometimes he doesn't understand even the statement because you don't understand the right-hand side and left-hand side of equations physicists write, for example. You have been at the Kavli IPMU for several years, interacting with physicists, so do you have any perspective to offer...?

Yukinobu Toda: Of course, I don't know anything about string theory, but sometimes I look at papers and some calculations and try to translate physics words into mathematics, say D-branes to sheaves or BPS states to stable objects. Then I have lots of things to learn from the physics side and lots of problems to solve, although I don't understand their physics origin. I also found that they are related to the classical problem in algebraic geometry.

Ooguri: You also attend string theory seminars. What do you gain by attending them and interacting with physicists?

Yukinobu Toda: I think there are many kinds of people in string theory. Some people's works are close to me, like Donaldson-Thomas invariants and derived category of coherent sheaves. In their seminars, I can learn something, but that is almost a seminar of mathematics.
Ooguri: A mathematician told me that physicists are like generating functions of conjectures. Some physicists are more useful for mathematicians than others. For example, Hiraku Nakajima was telling me that he particularly likes Edward's lectures, because even though he doesn't understand the motivations and where ideas come from, some of the statements Edward makes have sharp mathematical meanings to them, just like the equation that Tachikawa was quoting yesterday at the workshop, and these are something that mathematicians can work on.

Masahito Yamazaki: But then sometimes people want to know the logic behind it. I can make a statement that makes sense mathematically, and mathematicians can try to prove it. But they definitely want to know what's happening.

Witten: In any given case, I can't guarantee that there isn't a simpler answer. But the view of most physicists about many of the problems that we have been discussing is going to be that the best setting for these questions is in the quantum field theories that are important in physics.

\section{Quantum Entanglement}

Ooguri: We are still discussing what was happening in the 1990s. Now, we should move on to the new millennium. What do you think have been highlights in the past fourteen years?

Witten: Part of the answer is that the gaugegravity duality that was introduced by Maldacena is very deep. Even today people are still discovering interesting new facets of it. An important example was the work of Shinsei Ryu and Tadashi Takayanagi on entanglement entropy in gauge/gravity duality. They discovered a really interesting generalization of the Bekenstein-Hawking entropy of a black hole. Although I have not personally worked on this subject, the developments have been pretty interesting and may contain deeper clues about quantum gravity. If I could see the right way to do this, then I would probably work in this area myself, but at least so far I don't. But it's one of the things I'd recommend watching most closely.

Apart from Ryu and Takayanagi, I also would definitely recommend the papers of Horacio Casini, in some cases coauthored with Marina Huerta. One of these papers addressed the following question. A black hole has a Bekenstein-Hawking entropy. Roughly twenty years ago, Jacob Bekenstein considered the following question. Suppose an object falls into a black hole. The object has an entropy. When it falls into a black hole, its entropy disappears into the hole. The black hole gains mass when it absorbs the object, so its entropy goes up. The second law of thermodynamics says the total entropy should increase in this process, so in other words the black hole entropy increases by 
at least the entropy that the in-falling object had before approaching the black hole. This tells you basically that if an object has given energy and is small enough to fit inside a black hole of given mass, then there's an upper bound to its entropy. Bekenstein proposed such a bound, and people called it the Bekenstein bound, but for a long time no one could formulate precisely what this bound was supposed to say.

I am reminded here of what Fukaya said about the relationship between physics and mathematics, where he remarked that it can be hard to formulate precisely the terms that enter some of the statements made by physicists. In the case of the Bekenstein bound, the situation was as follows. In a situation in which the concepts (the size, energy, and entropy of the in-falling object) have clear meaning, the Bekenstein bound was trivially true and not very interesting. For example, consider a gas consisting of many particles bouncing around in a box. Here the size of the system and its energy and entropy all have a clear meaning. The Bekenstein bound was true but not very interesting because it was satisfied by a very wide margin. You could ask, could you find a situation where the Bekenstein bound is close to being saturated? You can accomplish this by considering not a whole gas of particles but a single particle in a box. More exactly, this gets close to saturating the Bekenstein bound if we can ignore the mass of the box, but that is an unrealistic assumption. To get close to saturating the Bekenstein bound, we really should consider a single particle that at a given time is almost certainly contained in a certain region in space-time even though there is no box keeping it there. (I say "almost certainly" because relativistic quantum mechanics does not permit us to say that a particle is definitely present in a given region.) Here for a single particle, we can define its energy, and we can identify (within general limits of relativistic quantum mechanics) the region that it is confined in, but it is hard to make sense of the entropy of a single particle. For a long time, there were many papers discussing this, many dozens and probably hundreds of papers, for the most part with limited insight. Then, there was a simple and quite brilliant paper by Horacio Casini, who showed that the right concept is entanglement entropy and that it can always be defined in a natural way and does enter in a universal Bekenstein-like bound. This paper was well ahead of the prevailing thinking in the field, and it was a number of years, I think, before people widely appreciated it.

Ooguri: For example, Casini's paper solved the species problem that I had been puzzled about for some time and gave a convincing explanation that it's not an issue.
Witten: There were what people thought were counterexamples to the Bekenstein bound, and so some people, and I was one, thought that if the bound was true, it was a statement about quantum field theories that can be coupled to gravity, not about all quantum field theories. But Casini showed that this was completely wrong. He gave a precise meaning to all the terms that entered the Bekenstein bounds, and he showed that it was a universal statement of quantum field theory that follows from general principles. That was extremely illuminating and like other work on entanglement entropy, one suspects it's probably an important clue, but it might take a younger person than I with fresh thinking to see what it is an important clue to.

I do want to mention one more contribution in that direction, which is by Casini and Maldacena with Rafael Bousso and Zachary Fisher (BCFM). Years ago, Bousso had formulated a covariant version of the Bekenstein bound; it is well adapted to problems in cosmology. Everything I have said about the Bekenstein bound has an analog for the Bousso bound. When you understood what it meant, it wasn't very interesting, and when it was interesting, you couldn't understand what it meant. In the recent work of BCFM, a precise formulation and proof of the Bousso bound are given, at least for quantum field theory in flat space-time.

Ooguri: I see that this new joint activity between quantum gravity and quantum information theory has become very exciting. Clearly entanglement must have something to say about the emergence of space-time in this context.

Witten: I hope so. I'm afraid it's hard to work on, so in fact, I've worked with more familiar kinds of questions. I have spent a lot of the last decade or so working on a succession of problems that probably were a little bit more out of the mainstream than most of my previous work. Also, I simply worked on these problems much longer than I usually worked on any one problem in the past. I guess the three problems that best fit what I have just said have been gauge theory and the geometric Langlands program, gauge theory and Khovanov homology, and superstring perturbation theory.

Superstring perturbation theory is best understood in terms of super Riemann surfaces. I should say that much of my knowledge about super Riemann surfaces comes from things I have learned from Pierre Deligne, both in the 1980s and more recently. Super Riemann surfaces are a fascinating generalization of ordinary Riemann surfaces to include odd or anticommuting variables. There is a fascinating algebro-geometric theory that was partly developed in the 1980s and not pursued so much since then. It would be great if it gets revived. By the way, we are having a workshop in May 2015 


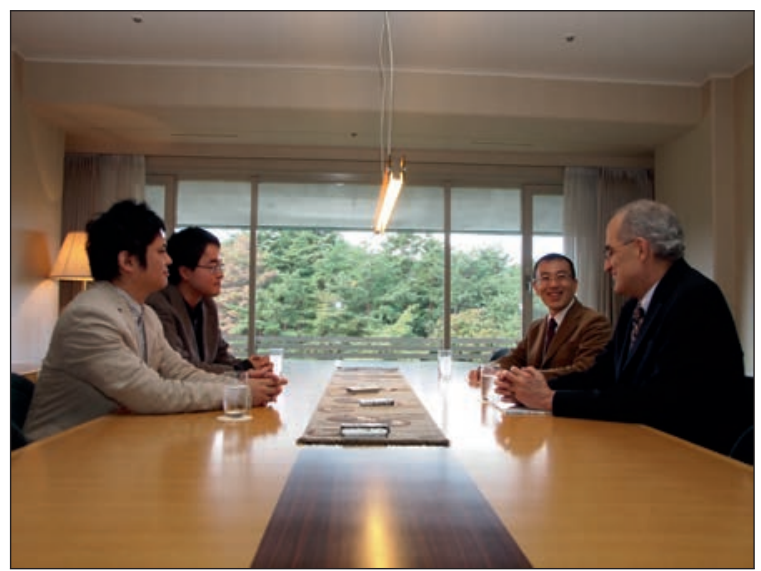

Toda, Yamazaki, Ooguri, and Witten.

at the Simons Center for Geometry and Physics in Stony Brook [Supermoduli, May 18-22, 2015], and algebraic geometers might be interested in it.

\section{Khovanov Homology}

Masahito Yamazaki: I was attending your lecture yesterday, and you were explaining how you came to the idea that Khovanov homology can be written as $N=4$ super Yang-Mills integrated over an unusual integration cycle. One thing that impressed me there was that your previous papers were the crucial input, namely your work with Anton $\mathrm{Ka}$ pustin in which you formulated the Kapustin-Witten equation, and also subsequent work you did with Davide Gaiotto on boundary conditions in $N=4$ super Yang-Mills theory. When you worked on these papers, did you already have in mind application to Khovanov homology?

Witten: The answer is "no": in those years, I knew about Khovanov homology and I was frustrated to not understand it, but I had no idea it was related to geometric Langlands. I was frustrated at not understanding Khovanov homology, because I felt that my work on the Jones polynomial ought to be a good starting point for understanding Khovanov homology, but I just could not see how to proceed. (From a mathematical point of view, Khovanov homology is a refinement or "categorification" of the Jones polynomial of a knot.) Actually, Sergei Gukov, Albert Schwarz, and Vafa had already given (in 2004) a physics-based interpretation of Khovanov homology, drawing in part on earlier work of Ooguri and Vafa. But I found it perplexing and a little frustrating that the relation of this to gauge theory was so indirect and remote. I wanted to find a more direct route, but for several years I found this difficult.

Eventually, however, some developments in the mathematical literature helped me understand that Khovanov homology should be understood using the same ingredients that are used to understand geometric Langlands. I didn't understand all of these clues, but I learned from two of them. One was the work of Dennis Gaitsgory on what mathematicians call quantum geometric Langlands (I am not sure this is the name a physicist would use) showing that the $q$ parameter of quantum geometric Langlands is related to the $q$ parameter of quantum groups and the Jones polynomial. The other was the work of Sabin Cautis and Joel Kamnitzer constructing Khovanov homology using a space of repeated Hecke modifications. I did not initially know what to make of those clues, but they were a sort of red flag hanging out there.

Hecke transformations are one of the most important ingredients in geometric Langlands. What they mean in terms of physics had bothered me for a long time and eventually had been the last major stumbling block in interpreting geometric Langlands in terms of physics and gauge theory. Finally, while on an airplane flying home from Seattle, it struck me that a Hecke transformation in the context of geometric Langlands is simply an algebraic geometer's way to describe the effects of a "'t Hooft operator" of quantum gauge theory. I had never worked with 't Hooft operators, but they were familiar to me, as they had been introduced in the late 1970s as a tool in understanding quantum gauge theory. The basics of how to work with 't Hooft operators and what happens to them under electric-magnetic duality were well known, so once I could reinterpret Hecke transformations in terms of 't Hooft operators, many things were clearer to me.

Cautis and Kamnitzer had interpreted Khovanov homology in terms of the B-model of a space of repeated Hecke transformations. Kamnitzer also conjectured in another paper that there would be an alternative description in terms of an A-model of the same space. Technically, it was hard to find the right A-model. I really wanted to understand the A-model, because that was the approach in which one could expect to achieve manifest three- or fourdimensional symmetry. My main goal in studying Khovanov homology was to find a description with manifest symmetry and a clear relationship to the gauge theory description of the Jones polynomial. I eventually succeeded in doing this. One of the trickiest elements was that the gauge fields have to obey a subtle boundary condition that I call the Nahm pole boundary condition. (The basic idea that leads to the Nahm pole boundary condition was introduced by Werner Nahm more than thirty years ago in his work on magnetic monopoles.) Luckily for me, I was familiar with the Nahm pole boundary condition and its role in electric-magnetic duality because of work that I had done with Davide Gaiotto a few years earlier. 
I suspect that the mathematics world could appreciate my work on Khovanov homology in the short to medium term and that the obstacle to this is largely a lack of familiarity with the Nahm pole boundary condition. With this in mind, I have been working with Rafe Mazzeo trying to give a detailed mathematical theory of that boundary condition. We have written one paper formulating rigorously the Nahm pole boundary condition in the absence of knots, and we are trying to generalize this to include the knots. The necessary inequalities are available, but some details are not yet in place.

Masahito Yamazaki: I see. That's a very nice story of the physics-mathematics interaction. You were partly motivated by the important papers in mathematics and interpreted them as a physicist. Then you have your own physics story and you are now trying to bring it back to mathematics.

Witten: As I have mentioned, the version that Cautis and Kamnitzer were actually able to understand was the B-model. Since it doesn't have manifest three-dimensional symmetry, I decided to concentrate on the A-model, but if I ever have a couple of months to spare, I would try to explain as a physicist the Cautis and Kamnitzer B-model. I'm reasonably optimistic I could do that and I think it would be illuminating. The only problem is that there are a lot of things like that-interesting loose ends that I think I could clarify if I spend a few months on them.

\section{Langlands Correspondence and Gauge Theory Dualities}

Ooguri: That the Langlands correspondence has something to do with $S$-duality was there even in the late 1970s. When was it that you actually realized the significance of it?

Witten: I didn't give the complete explanation of my interaction with Michael Atiyah in 1977. He told me about two things that were new to me. One was the Montonen-Olive paper and the other was the Langlands correspondence, which plays a central role in number theory but which I had never heard of. He had noticed that the dual group of Langlands and the dual group that enters the Montonen-Olive conjecture (and which had been introduced earlier by Peter Goddard, Jean Nuyts, and Olive) were the same. On this basis, Atiyah suspected that the Langlands correspondence has something to do with the Montonen-Olive conjecture.

Ooguri: So that was in the late 1970s?

Witten: It was December of 1977 or January of 1978. That was when I visited Oxford for the first time.

Ooguri: Did you take seriously already at that time that the Langlands correspondence had something to do with this gauge theory dynamics?
Witten: Well, I didn't forget about it, but since, as I already told you, I was skeptical about MontonenOlive duality, I didn't seriously try to relate it to Langlands duality and I didn't try to learn what Langlands duality was. I did not learn anything more about these matters until the late 1980s. Then I learned just superficially about the Langlands correspondence. If one knows even a little bit about the Langlands correspondence and a little bit about conformal field theory on a Riemann surface, one can see an analogy between them. I wrote a paper that was motivated by that, but then I realized that my understanding was too superficial to lead to anything deep, so I abandoned the matter for a number of years.

Ooguri: I remember when I was a postdoc at the Institute for Advanced Study in 1988 and 1989, Robert Langlands himself was actually quite interested in conformal field theory. I am not sure exactly which aspect he was interested in, however.

Witten: I don't think he was motivated by the Langlands correspondence. But I think his work was influential. Even though in a sense he didn't precisely make any major breakthrough himself, he helped to find the questions that stimulated the later development of Stochastic Loewner Evolution, which has had a major impact on mathematics and has even enlightened physicists about new ways to think about some questions in conformal field theory. I think Langlands was an influence behind this work, but I do not believe his interest in conformal field theory was motivated by the Langlands correspondence or by gauge theory dualities. This is my impression from interacting with him over the years.

As I have already remarked, in the late 1980s, after spending some time trying to develop the analogy between conformal field theory and the Langlands correspondence, I concluded reluctantly that the analogy in the form I was developing was way too superficial, so I stopped. But then around 1990, I heard about new work of Alexander Beilinson and Vladimir Drinfeld on the geometric Langlands correspondence. This had a few consequences. First of all, it confirmed that my understanding of what the duality would mean in physics was way too superficial. What they had was much more incisive and much more detailed than my rather primitive analogy between the Langlands correspondence and conformal field theory. Their work confirmed that physics that I knew was relevant. But I was troubled, because they were using conformal field theory in a way that didn't make any sense to me. They studied conformal field theory at negative integer levels-in physics positive integers are more natural here-and used it in ways that looked quite strange. 
Familiar Ingredients That Seemed Not to Fit

As I explained yesterday in my lecture at the Kyoto Prize Symposium, for a number of years, the "volume conjecture" concerning the Jones polynomial (formulated and developed starting around 2000 by Rinat Kashaev, Hitoshi Murakami, and Jun Murakami, among others, and explained to me in large part by Sergei Gukov) bothered me. Although their statements bore a superficial resemblance to physically well-motivated statements - in fact, to statements that I myself had made in my original paper on the Jones polynomial in 1988-there was a crucial difference. They seemed to have complex critical points that made exponentially large contributions, and this normally is not possible in physics. I am not sure if this point bothered anyone else, but it bothered me. It turned out that this was a good question to think about, since I eventually found a nice explanation, and this was a turning point in enabling me to understand Khovanov homology via gauge theory.

The work of Beilinson and Drinfeld on geometric Langlands bothered me in much the same way. They were using familiar ingredients of physics, but they were using them in ways that did not seem to fit. It looked like somebody had taken a bunch of chess pieces, or perhaps here in Japan I should say a bunch of shogi pieces, and placed them on the board at random. The way that the pieces were arranged did not make any sense to me. That bothered me, but I could not do anything about it.

Actually, the very little bit of what Beilinson and Drinfeld were saying that I could understand made me wonder if the work of Nigel Hitchin would be relevant to them, so I pointed out to them Hitchin's paper in which he had constructed commuting differential operators on the moduli space of bundles on a curve. Differently put, Hitchin had in a certain sense quantized the classical integrable system that he had constructed a few years before. Although I understood scarcely anything of what Beilinson and Drinfeld were saying, I did put them in touch with Hitchin's work, and actually, in their very long, unpublished foundational paper on geometric Langlands that you can find on the Web, Beilinson and Drinfeld acknowledged me very generously, far overestimating how much I had understood. All that had really happened was that based on a guess, I told them about Hitchin's work, and then I think that made all kinds of things obvious to them. Maybe they felt I knew some of those things, but I didn't. But anyway, there were ample reasons in those years to think that geometric Langlands had something to do with physics, but as you can see I still couldn't make any sense out of it.

Ooguri: So, what inspired you to return to this?
Witten: A decade later there was a workshop at the Institute for Advanced Study on geometric Langlands for physicists. Were you there?

Ooguri: I was invited, but there was a conflict of schedule, so I couldn't go. I missed it.

Witten: There were two long series of lectures and then there were a couple of outliers. The long series were very well done, but they did not help me very much. Mark Goresky gave a long series of lectures aiming to tell physicists what is the Langlands correspondence. The only trouble for me was that to the extent that one can explain this topic in a couple of lectures, assuming essentially no knowledge of algebra beyond the definition of a field (in the algebraic sense), I was familiar with the Langlands correspondence already. Namely, I didn't really know anything about it, but I knew as much as one could explain in a few hours starting from zero. So I couldn't really get much out of those lectures.

In addition, Ed Frenkel (who had been the prime mover behind the occurrence of this workshop) gave a series of lectures that, as far as I was concerned, were basically about the shogi board on which the pieces have been arranged at random. I really couldn't get much out of those lectures either, because I already knew that people working on the geometric Langlands were taking familiar pieces from the shogi set and arranging them on the board at random as far as I was concerned.

There were a couple of additional lectures that weren't part of any series. One of them was by David Ben-Zvi. He told us about what was supposed to be an approximation to the geometric Langlands correspondence. I think he was talking largely about the work of another mathematician, Dima Arinkin. What was supposed to be the approximation to the geometric Langlands correspondence was Tduality on the fibers of the Hitchin fibration. This was described by Ben-Zvi in a complex structure in which the fibers of the Hitchin fibration are holomorphic, so the T-duality is a holomorphic duality. It was already known to physicists that the T-duality on the fibers of the Hitchin fibration comes from Montonen-Olive duality in four dimensions, and of course ever since Atiyah's observations of 1977-78, I had been aware of the possibility that some version of the Langlands correspondence might be associated to Montonen-Olive duality. But what about the fact that Ben-Zvi was only claiming to deduce from T-duality an approximation to geometric Langlands duality rather than the real thing? At a certain point, I started to suspect that the reason for this was simply that Ben-Zvi was describing the situation in the wrong complex structure. The idea was that the same T-duality of Hitchin's moduli space, viewed differently, would give a mirror symmetry between a B-model in a 
certain complex structure and an A-model in a certain symplectic structure. This mirror symmetry was supposed to be the true geometric Langlands duality, not the approximation. Actually, the reason I started working on geometric Langlands with Anton Kapustin was that he had studied generalized complex geometry in two-dimensional dualities. In that world, a family of dualities can degenerate, and a mirror symmetry can degenerate to a holomorphic duality.

When one starts thinking along these lines, it soon makes a lot of sense that the geometric Langlands duality is really a mirror symmetry, which can degenerate to a holomorphic duality, and that this is the approximation Ben-Zvi taught us about. I became convinced that that had to be right. There were still a few hurdles to overcome. The most difficult one I already described earlier. One does not get to first base with the Langlands correspondence without Hecke operators, so it was necessary to have a physical interpretation of Hecke operators in terms of 't Hooft operators of gauge theory. It was also necessary to know how to interpret the A-model of the cotangent bundle of a complex manifold $M$ in terms of differential operators on $M$. This actually was fairly close to things that Kapustin had done earlier. Once these points were understood, it was pretty clear to me as a physicist what geometric Langlands duality is.

\section{Felt Like I Discovered the Meaning of Life and Couldn't Explain It}

But it was very hard to write a paper about it. It took about a year. For that year, I felt like someone who had discovered the meaning of life and couldn't explain it to anybody else. And in a sense, I still feel that way for the following reason. Physicists with a background in string theory or gauge theory dualities can understand my paper with Kapustin on geometric Langlands, but for most physicists this topic is too detailed to be really exciting. On the other hand, it is an exciting topic for mathematicians but difficult to understand because too much of the quantum field theory and string theory background is unfamiliar (and difficult to formulate rigorously). That paper with Kapustin may unfortunately remain mysterious to mathematicians for quite some time.

Masahito Yamazaki: Maybe that means that we have to wait an extra ten or fifteen years before...

Witten: We indeed may have to. I think it's actually very difficult to see what advance in the near term could make the gauge theory interpretation of geometric Langlands accessible for mathematicians. That's actually one reason why I'm excited about Khovanov homology. My approaches to Khovanov homology and to geometric Langlands use many of the same ingredients, but in the case of

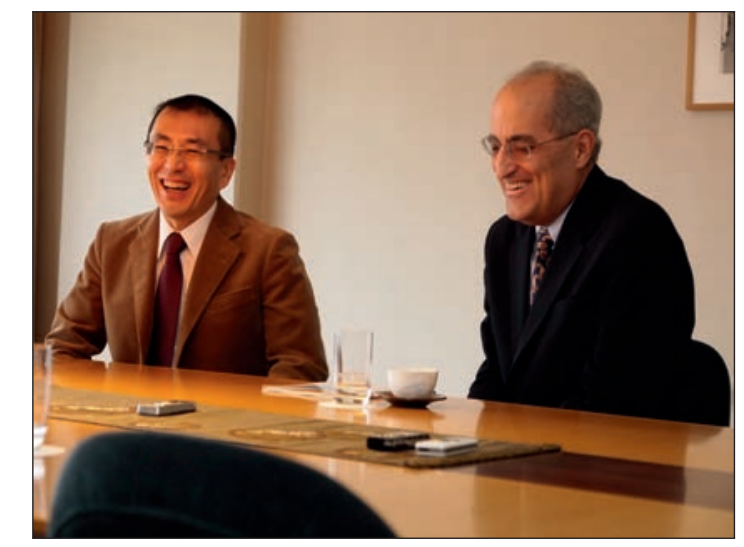

Ooguri and Witten.

Khovanov homology, I think it is quite feasible that mathematicians could understand this approach in the near future if they get excited about it. I believe it will be more accessible. If I had to bet, I think I have a decent chance to live to see gauge theory and Khovanov homology recognized and appreciated by mathematicians, and I think I'd have to be lucky to see that in the case of gauge theory and the geometric Langlands correspondence-just a personal guess.

Yokinobu Toda: Do you think your idea of the $S$-duality and the geometric Langlands can be somehow applied to the honest Langlands program?

Witten: I see that as being far away. For me personally, it's a dream that eventually number theory would make contact with physics sometime, but I doubt it will be soon.

There are all kinds of areas where specific number theory formulas appear in physics, and these may be clues that the dream will come true one day. But to really get me excited, somehow the number theory would have to enter the physics in a more structural way. I'm not that interested in a specific formula that comes out of a physics calculation in a more or less ad hoc fashion. Number theory would have to be more integrated with the physics to get me excited, and I don't see that happening soon.

In my work, I concentrated on the geometric form of the Langlands correspondence because I could see that there was hope to really understand it in the context of the physics-based tools that were at hand. There might be something like that one day for the Langlands correspondence of number theory, but probably a lot is missing, and we do not know what has to happen first. I feel that the reason I was able to make progress was that my focus was much more narrow than trying to understand the Langlands correspondence of number theory. 
Yokinobu Toda: The relationship between $S$ duality and geometric Langlands was surprising to me, as number theory seems to be a research area far from physics.

Witten: Nevertheless, there have been many developments which one day may be seen as important clues. One of the deepest was started roughly fifteen years ago by Savdeep Sethi and Michael Green and then continued by Green with many collaborators. In the original work, Sethi and Green were trying to understand certain low energy $R^{4}$ interactions in Type IIB superstring theory in ten dimensions (here $R$ is the Riemann tensor). They made an amazing discovery, I would say: the answer is given by a certain nonholomorphic Eisenstein series of weight $3 / 2$. Although my knowledge of number theory is very superficial, I think that this sort of thing is much closer to the interests of modern number theorists than the aspects of classical modular forms that usually appear in two-dimensional conformal field theory.

Ooguri: Those objects which are not totally modular have also appeared in number theory.

Witten: That is correct. A lot of things that number theorists like have appeared in physics, and some have even appeared in my own work. Plenty has been found to show that the physics theories that we work on as string theorists are interesting in number theory. These theories know something about number theory, but personally I don't see an opportunity to really make contact in a structural way with number theory in the foreseeable future. I can't even formulate what it would mean to make such contact, so I can't even properly tell you what we can't do but I think the time is not right to do it.

Anyway, that's why I personally concentrated on geometric Langlands rather than on number theory, and geometric Langlands was hard enough. It was a lot of work to understand it, but I think that having understood it, many things that mathematicians do involving geometric aspects of representation theory are much more accessible as part of physics. For example, I did not understand what Hiraku Nakajima explained yesterday at the Kyoto Prize Workshop, but I think that an understanding might involve some of the things that were clear after working with geometric Langlands. I can't promise, but it is worth a try.

Just one obvious thing is that although Nakajima did not have time to explain the whole picture, at the end of his lecture he was telling us about the affine Grassmannian. Isomorphism classes of 't Hooft operators are associated to cycles in the affine Grassmannian, so if a mathematician tells you about the affine Grassmannian, you probably want to think about at least part of what you are hearing in terms of 't Hooft operators. I can make no promises, but I feel it would definitely be worth a try to understand what Nakajima was saying from a physicist's viewpoint.

I am sure, at any rate, that there is much more that can and should be done to understand much more of geometric representation theory from a physical viewpoint. In fact, part of the original work of Beilinson and Drinfeld on geometric Langlands has still not been understood to my satisfaction. Here I have in mind the use of conformal field theory at what they call the critical level (level $-h$, where $h$ is the dual Coxeter number) to construct the A-model dual of certain B-branes (the ones that are associated to opers, in the language of Beilinson and Drinfeld). Davide Gaiotto and I obtained a few years ago a reasonable understanding of what electric-magnetic duality does to the variety of opers, but I still do not really feel I understand its relation to conformal field theory. However, in the last few years physicists working on supersymmetric gauge theories in four dimensions and their cousins in six dimensions have made several discoveries involving the role of conformal field theory at the critical level, so the time may well be right to resolve this point.

\section{How to Work with Mathematicians}

Yokinobu Toda: I have a general question. What kinds of problems should mathematicians work on?

Witten: Well, there are lots of problems that algebraic geometers study that involve dualities studied by physicists. In many of those cases, I will not be able to give much advice, as I am not an expert on recent developments. In some cases, I am still struggling to understand things that physicists did quite some time ago that are very relevant. Just to give one example, the Gopakumar-Vafa and Ooguri-Vafa formulas have been very influential for algebraic geometers, but as a physicist, I was never satisfied that I understood them. So I actually spent a lot of time in the last year with a student (Mykola Dedushenko) trying to understand these formulas better. In this work, I was doing some of the homework that I'd have to understand before even trying to answer your question.

Ooguri: You will talk about it next week at the Kavli IPMU [later published as a paper, "Some details on the Gopakumar-Vafa and Ooguri-Vafa formulas," arXiv:1411.7108].

Witten: Going back to Yukinobu's question, although there are many areas of current interest on which I probably cannot give useful advice, there is one bit of advice that I actually would offer to algebraic geometers. I do recommend super Riemann surfaces. I'm sure there's a deep theory there. I can't promise anything about how quickly it will emerge. A deep theory will probably only be developed in the near term if enough people 
get excited about it. Maybe the workshop we are having next spring at the Simons Center will help make that happen. We will see.

Ooguri: It's certainly true that when people were working on the finiteness and vanishing of the cosmological constant in perturbative string theory twenty-five to thirty years ago, it was not totally satisfactory. The complete understanding came only after your proper description in terms of geometry of super Riemann surfaces.

Witten: Thank you, Hirosi, and I'm glad you think so. Not all physicists agree, because it is possible to express everything in terms of picturechanging operators and so on, hiding the super Riemann surfaces. I think personally that when one does that, one doesn't understand properly what the formulas mean. But not everybody agrees.

I think one reason that the theory of super Riemann surfaces stopped developing in the 1980s was that physicists became satisfied with their partial understanding in which the super Riemann surfaces were hidden. There is a tremendous beauty to this subject that I think is simply missed if you try to understand things that way. I care about it enough to have spent several years by now on spelling out details of the description in terms of super Riemann surfaces.

It has seemed unclear that a lot of physicists would really get excited about the sort of details that I was trying to fill in. So one of my hopes has been that mathematicians will get excited about developing super Riemann surface theory. I can't promise, but I think they should.

Ooguri: Do you expect also new physics insight coming out of the more precise understanding of perturbative string theory?

Witten: The answer may depend on what you mean by physics insight. I think that one understands better what superstring perturbation theory means if one formulates it in terms of integration on the moduli space of super Riemann surfaces. That is insight of a sort. However, I do not see any evidence at the moment that incorporating super Riemann surfaces in the way that we understand perturbation theory will help us with nonperturbative questions, for example, or with understanding better the symmetry structure of string theory or whatever may be the correct concept.

Masahito Yamazaki: Let me ask my last question. You're working partly in the area of mathematical physics. You have a lot of discussion with mathematicians and also write math papers.

Witten: Well, I write math papers in very special cases where I think something I could actually do would be illuminating. Recent examples have been my work with Ron Donagi on some foundational questions about the moduli space of super Riemann surfaces and the work with Rafe Mazzeo that I mentioned before on the Nahm pole boundary condition.

Masahito Yamazaki: I see. So, my question is, what's the advice if a physicist wants to work with a mathematician effectively?

Witten: It's really difficult to give advice. Usually producing rigorous proofs requires very detailed methods. That makes it hard for a physicist, and so I myself have only done that in very special cases where I thought something was really missing that was actually simple enough that I could help do it if I had the right collaborator. Some physicists would want to go into more detail and learn the techniques for rigorous proofs in a particular area, but most physicists I think will only be happy and successful doing that in very special cases like the ones I've picked.

Masahito Yamazaki: I see. Is it also true that in many of your works, the conversation with some mathematicians has been an inspiration for you?

Witten: This usually happens when something a mathematician has done involves an aspect of the physics that hasn't been understood and that doesn't make sense to me. I mentioned earlier one case involving the volume conjecture. For years I could not understand the results in this area because complex critical points were making exponentially large contributions. I kept putting it aside, not able to make progress.

Finally, in the summer of 2009, I attended a conference at the Hausdorff Institute in Bonn on the twentieth anniversary of the Chern-Simons theory. I heard more lectures on the volume conjecture. To me, it was just embarrassing to not understand why exponentially large contributions were coming in. I feel vindicated in hindsight for worrying so much about this question, because the answer turned out to be really useful.

Masahito Yamazaki: I see. In that case the feeling that the pieces are not in the right place led you to the question, which you eventually solved, and also it led to new developments.

Witten: Yes. Another case was when I felt that Beilinson and Drinfeld had the shogi pieces jumbled on the chessboard.

\section{Message for Young Students}

Ooguri: Pieces are placed in a wrong way, but if you look at it in different dimensions, perhaps they are totally aligned.

I also have a final question. In the interview with Tohru Eguchi twenty years ago in Sugaku Seminar, he asked you about the prospects at the interface of mathematics and physics, and you replied saying that the area had been certainly growing very strongly and you predicted that the progress would continue in the foreseeable future. Certainly 


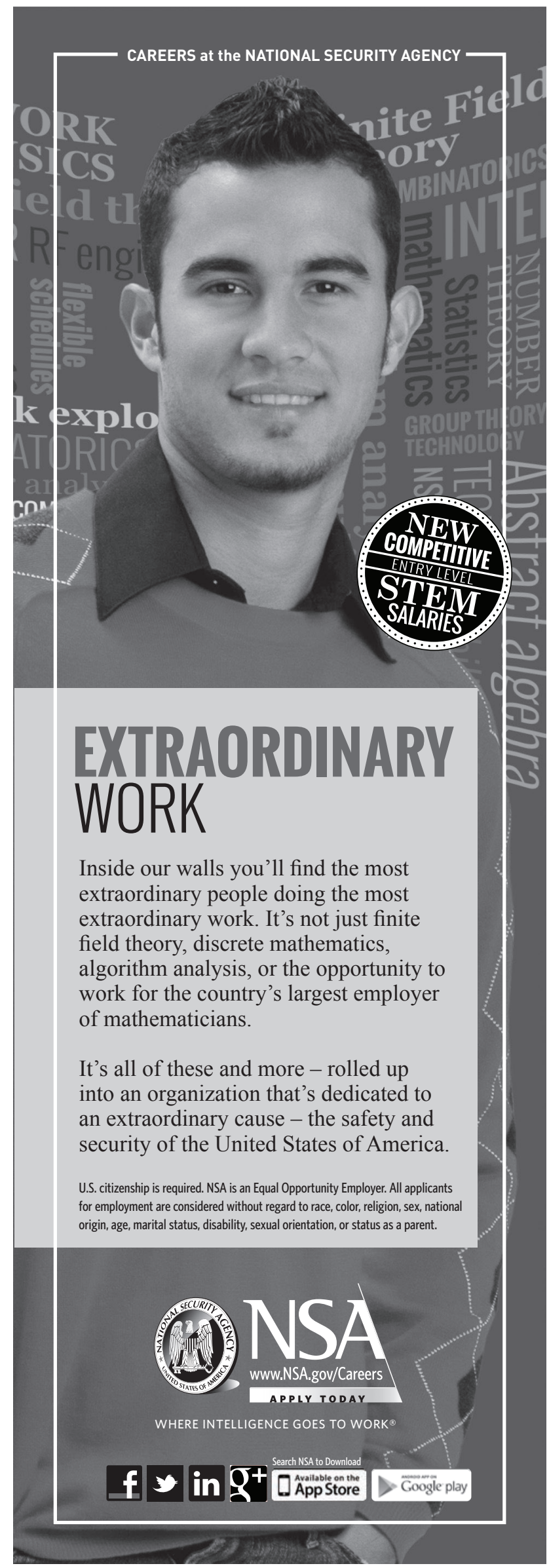

your prediction has been amply verified in the last twenty years. Given this, my question is, again, what is your prospect for the next twenty years? Can you also give advice on the future of the field for young students who will be reading this article?

Witten: First of all, in the last twenty years, not only has this interaction of math and physics continued to be very rich but it has developed in such diversity that very frequently exciting things are done which I myself am able to understand embarrassingly little about, because the field is expanding in so many directions.

I am sure that this is going to continue and I believe the reason it will continue is that quantum field theory and string theory, I believe, somehow have rich mathematical secrets. When some of these secrets come to the surface, they often come as surprises to physicists because we do not really understand string theory properly as physics-we do not understand the core ideas behind it. At an even more basic level, the mathematicians are still not able to fully come to grips with quantum field theory and therefore things coming from it are surprises. So for both of those reasons, I think that the physics and math ideas generated are going to be surprising for a long time.

I think there are definitely exciting opportunities for young people to come and help explain what it all means. We don't understand this properly. We got a wider perspective in the 1990s when it became clear that the different string theories are unified by nonperturbative dualities and that string theory in some sense is inherently quantum mechanical. But we're still studying many different aspects of a subject whose core underlying principles are not clear. As long as that is true, there are opportunities for even bigger discoveries by today's young people. But if I could tell you exactly what direction you had to go in, I would be there.

Ooguri: Thank you very much for taking time to talk to us. It has been fun. Congratulations again on your Kyoto Prize.

Witten: Thank you so much for your kind words on the Kyoto Prize, and also thank you for the discussion, because the discussion has helped me remember how much we have advanced in the last twenty years.

Ooguri: Let's meet again twenty years from now to assess our progress in the next twenty years.

Witten: Let's try. For that we will all have to exercise and keep fit. 